\title{
Using CHIMERA detector at LNS for gamma-particle coincidences
}

G. Cardella $^{1}$, L. Acosta $^{9}$, L. Auditore ${ }^{4}$, M.B. Chatterjiee $^{8}$, A. Castoldi ${ }^{7}$, E. De Filippo ${ }^{1}$, D. Dell'Aquila ${ }^{6}$, S. De Luca ${ }^{4}$,

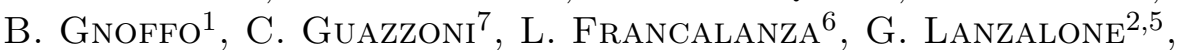
I. Lombardo ${ }^{6}$, N. Martorana ${ }^{2,3}$, S. Norella ${ }^{4}$, A. Pagano ${ }^{1}$, E.V. Pagano ${ }^{2,3}$, M. PaPA ${ }^{1}$, S. Pirrone ${ }^{1}$, G. Politi ${ }^{1,3}$, L. Quattrocchi ${ }^{4}$, F. Rizzo ${ }^{2,3}$, P. Russotto ${ }^{1}$, A. Trifirò $^{4}$, M. Trimarchi ${ }^{4}$, G. Verde $^{1}$ and M. Vigilante ${ }^{6}$

${ }^{1}$ INFN, Sezione di Catania, Via S. Sofia, 95123 Catania, Italy 2 INFN - Laboratori Nazionali del Sud, Via S. Sofia, Catania, Italy

${ }^{3}$ Dip. di Fisica e Astronomia, Università di Catania, Via S. Sofia, Catania, Italy

${ }^{4}$ INFN - Gruppo collegato di Messina, Dip. di Fisica, Università di Messina, Italy

${ }^{5}$ Facoltà di Ingegneria e Architettura, Università Kore, Enna, Italy

${ }^{6}$ INFN, Sezione di Napoli, Dipartimento di Fisica, Università Federico II, Napoli, Italy

${ }^{7}$ Politecnico di Milano, Dipartimento di Elettronica, Informazione e Bioingegneria and INFN, Sezione di Milano, Italy

${ }^{8}$ Saha Institute for Nuclear Physics, Kolkata, India

${ }^{9}$ Instituto de Física, Universidad Nacional Autonoma de Mexico, Mexico D.F. 0100, Mexico

\begin{abstract}
We have recently evaluated the quality of $\gamma$-ray angular distributions that can be extracted in particle-gamma coincidence measurements using the CHIMERA detector at LNS. $\gamma$-rays have been detected using the $\mathrm{CsI}(\mathrm{Tl})$ detectors of the spherical part of the CHIMERA array. Very clean $\gamma$-rays angular distributions were extracted in reactions induced by different stable beams impinging on ${ }^{12} \mathrm{C}$ thin targets. The results evidenced an effect of projectile spin flip on the $\gamma$-rays
\end{abstract}


angular distributions. $\gamma$-particle coincidence measurements were also performed in reactions induced by neutron rich exotic beams produced through in-flight fragmentation at LNS. In recent experiments also the Farcos array was used to improve energy and angular resolution measurements of the detected charged particles. Results obtained with both stable and radioactive beams are reported.

\section{Introduction}

Relatively large yields of various neutron rich and poor exotic beams, of energy ranging from 20 to $50 \mathrm{MeV} / \mathrm{A}$, are available at LNS [1], and in particular we used ${ }^{6,8} \mathrm{He},{ }^{8,9} \mathrm{Li},{ }^{10,11} \mathrm{Be},{ }^{13} \mathrm{~B},{ }^{16,17} \mathrm{C},{ }^{68} \mathrm{Ni}$. In order to benefit of such beams with CHIMERA detector [2,3] a tagging system [4] was developed to identify event by event the isotopic composition of the produced exotic particles. This system was recently updated to allow trajectory measurement with a PPAC detector. To improve the energy and angular resolution measurements of particles produced in reactions induced by the exotic beams we used a prototype of the new FARCOS detection system under construction in Catania [5]. Moreover, as recently shown [6], it was possible to extract further information on reaction mechanisms also by looking to $\gamma$-ray energy and angular distribution by using the $\operatorname{CsI}(\mathrm{Tl})$ detectors of the CHIMERA sphere (from $30^{\circ}$ to $174^{\circ}$ ). This latter information will be of fundamental interest in one of the next experiments planned to be performed in CHIMERA, i.e. the study of isoscalar excitation of the PIGMY resonance on ${ }^{68} \mathrm{Ni}[7]$. In this paper we shortly describe in the next paragraph the production and identification of the fragmentation beam. In the last paragraph we summarize some of the recently published performances of our $\mathrm{CsI}(\mathrm{Tl})$ crystals when they are used as $\gamma$-ray detectors and some results concerning the coincidences between particles and $\gamma$-rays, together with future perspectives.

\section{Beam production, and tagging system}

The beam is produced by fragmentation reactions, using the beams accelerated by the superconducting Cyclotron (CS) of the Laboratori Nazionali del Sud in Catania. A Beryllium target is placed, close to the CS output extraction channel. The standard primary beam intensity, up to now used, is of the order of 100 Watts. The beam selection is obtained with appropriate setting of two $45^{\circ}$ dipoles of the main beam transport line of the CS. 


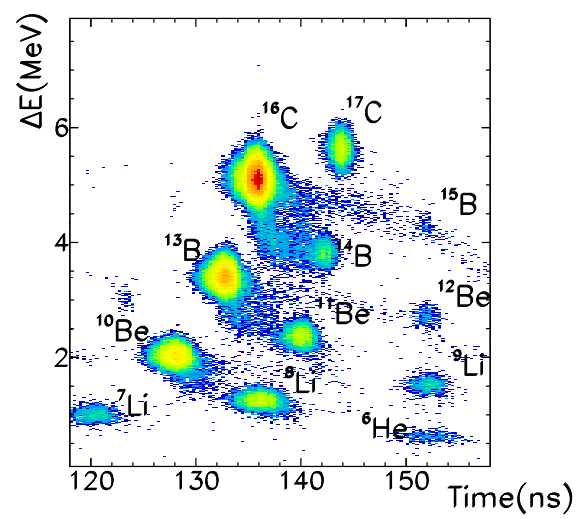

Figure 1: Identification scatter plot for the production of ${ }^{16} \mathrm{C} /{ }^{10,11}$ Be beams.

The tagging system is based on the use of a $32 \times 32$ strips double side silicon strip detector (DSSSD) measuring the energy loss of the impinging beam particles. The identification of the particles is achieved by combining this energy loss with the time of flight information (TOF). The start of this TOF measurement is provided by a large size micro-channel plate (MCP) [4] mounted $13 \mathrm{~m}$ away from the DSSSD. The stop is given by the signal delivered by the front strips of the DSSSD.

An example of the obtained identification scatter plot is shown in fig.1 for the production of ${ }^{16} \mathrm{C}$ and ${ }^{10,11} \mathrm{Be}$ by the fragmentation of ${ }^{18} \mathrm{O} 55 \mathrm{AMeV}$ primary beam. To measure trajectories we have installed, at the entrance of the CHIMERA sphere, a PPAC position sensitive detector. The position information provided by DSSSD and PPAC allows to extract the beam trajectory. This is a basic measurement to sketch the beam profile at the target position during the beam commissioning phase.

\section{Gamma ray detection}

In reactions with radioactive beams, due to the low beam intensity, it is today mandatory to perform measurements as complete as possible. It is for instance very important to be able to detect the $\gamma$-rays in coincidence with particles emitted in the reaction. With CHIMERA we detect and identify $\gamma$-rays in all $\mathrm{CsI}(\mathrm{Tl})$ detectors. However we have a better signal to noise ratio in the detectors of the spherical part due to kinematical focusing, and 


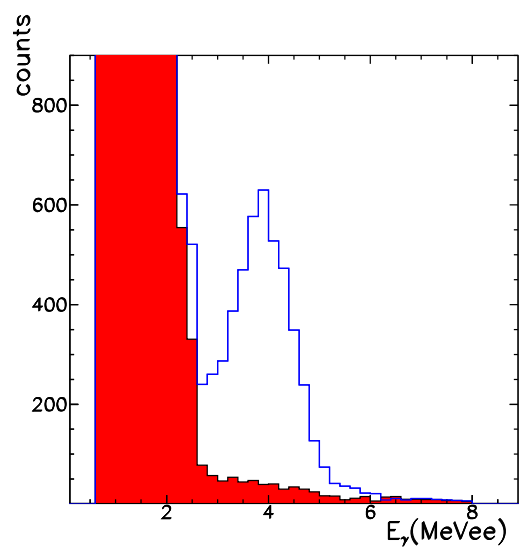

Figure 2: $\gamma$-ray spectrum detected in CHIMERA CsI(Tl) in coincidence with proton from elastic scattering on carbon target (filled spectrum), and in coincidence with proton from inelastic scattering leaving the carbon excited at the $4.44 \mathrm{MeV}$ level (empty spectrum).

to the larger solid angle of the detectors. We measured the CsI(Tl) response function to $\gamma$-rays, looking to the detection of the well know $4.44 \mathrm{MeV} \gamma$ ray, emitted by the decay of the first excited level of ${ }^{12} \mathrm{C}$. This state can be simply excited using proton scattering. In such kind of reactions one can look for the detection of this $\gamma$-ray triggering the event with the inelastic scattered proton. This is a way to measure also the detector efficiency. In fig.2 we show the spectrum collected in one ring of the sphere, in coincidence with such inelastic scattering events (empty spectrum). Background can be evaluated collecting the same number of events, triggered by the detection of protons from elastic scattering (full spectrum). One can see that the peak to noise ratio is rather good. Resolution is of the order of $0.5 \mathrm{MeV}$, as can be deduced by the missing observation of the first and second escape peaks. From comparison with GEANT-IV simulations, one understand that the energy response function is centered on the first escape peak at about 3.9 MeVee. We have also verified that we can simply extract angular distributions from such events. They are very useful to understand the multipolarity of the emitted $\gamma$-ray, so obtaining information about the spin of the observed resonance. It is useful to obtain information about the multipolarity of the emitted $\gamma$-ray, in such a way the spin of the observed resonance can be evaluated. For instance, in fig. 3 the CM angular distributions of $\gamma$-ray obtained at different proton beam energy from the decay 
of the $4.44 \mathrm{MeV}$ level is reported. The angular distribution is described by the formula $\sin ^{2}(2 \theta)+$ cost as it is expected, for quadrupole decay, in case of proton spin-flip events [6]. Similar results were obtained for all investigated energies, more details can be found in ref. [6]. A pure $\sin ^{2}(2 \theta)$ behavior is obtained using a zero spin projectile as ${ }^{16} \mathrm{O}$.

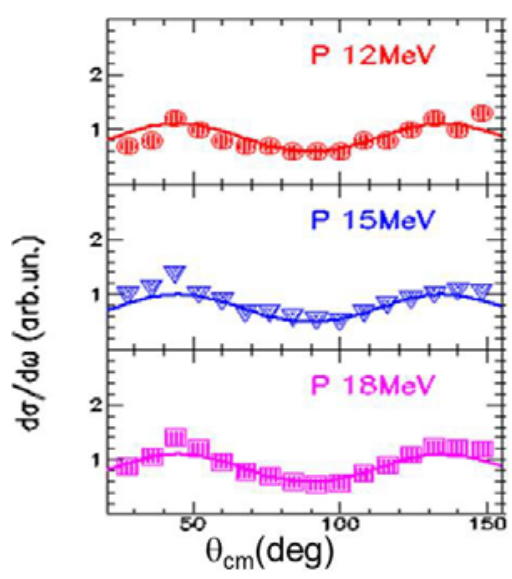

Figure 3: Center of mass angular distributions of $\gamma$-ray from the decay of ${ }^{12} \mathrm{C}^{4.44}$ populated at different proton beam energies.

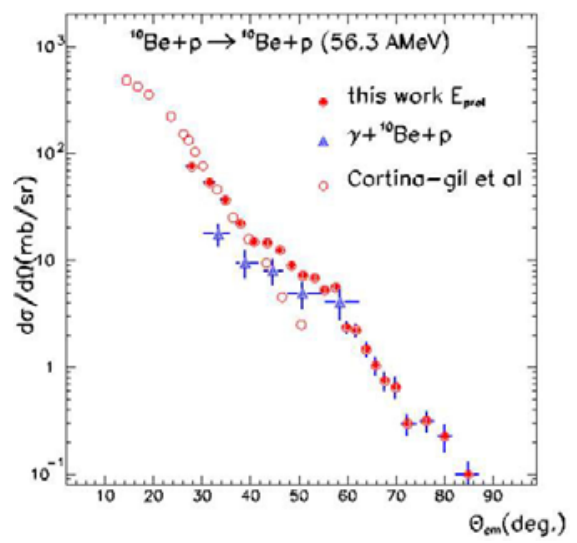

Figure 4: Center of mass angular distributions measured for the reaction ${ }^{10} \mathrm{Be}+\mathrm{p}$, see text.

It is well known that $\gamma$-rays can be used as a signature of inelastic channels. In fig. 4 we show how this effect is observed with CHIMERA. In this figure we plot as filled dots the elastic scattering angular distribution mea- 
sured for the reaction ${ }^{10} \mathrm{Be}+\mathrm{p}$ at $56 \mathrm{~A} . \mathrm{MeV}$. This angular distribution was extracted with the kinematical coincidence method that we have recently developed [8]. Comparing such angular distribution with the one measured in the past by Cortina-Gil [9] with SPEG (open dots), we note that our data have the same behavior of those obtained with spectrometer up to about $35^{\circ}$. At larger angles we observe a change in the slope of the angular distribution. This is due to the contribution of inelastic channels that cannot be discriminated by the elastic one in our data, due to the resolution of our $\mathrm{CsI}(\mathrm{Tl})$ detector. This effect is shown by the inelastic angular distribution (blue triangles) obtained measuring in coincidence with $\gamma$-ray the couples of proton and ${ }^{10} \mathrm{Be}$.

Further work is in progress to improve the energy resolution of $\mathrm{CsI}(\mathrm{Tl})$ detectors by using the new GET electronics [10]. The new electronics will also add new trigger capabilities for the $\mathrm{CsI}(\mathrm{Tl})$ stage that at present time is not available with standard DAC of CHIMERA, where the main trigger is given by electronic signals delivered by silicon detectors.

\section{References}

[1] see http://fribs.lns.infn.it/upgrade-results.html

[2] A.Pagano et al Nucl.Phys. A 734 (2004) 504 and ref. therein

[3] A.Pagano, Nuclear Physics News International, 22:1(2012)25

[4] I. Lombardo et al Nucl.Phys.B (Proc.Suppl.) 215(2011)272

[5] G.Verde et al Journ. of Phys.: Conf. Ser. 420 (2013) 012158; E.Pagano et al proceedings of this conference; Technical design report -TDR/INFN for the farcos project (https://192.84.151.50/joomla/)

[6] G.Cardella et al NIMA 799(2015)64

[7] E. G. Lanza, A. Vitturi, M. V. Andres, F. Catara and D. Gambacurta, Phys. Rev. C 84, 064602 (2011)

[8] L.Acosta et al NIM A 715 (2013) 56

[9] Cortina-Gil et al Phys.Lett.b 401(1997)9

[10] E.Pollacco et al Physics Procedia, Volume 37, 2012, Pages 1799 